\title{
Fibroblast growth factor-21 is a potential diagnostic factor for patients with gestational diabetes mellitus
}

\author{
CHENGFANG XU, ZHENYAN HAN, PING LI and XUEJIAO LI \\ Department of Obstetrics and Gynecology, The Third Affiliated Hospital, Sun Yat-sen University, \\ Guangzhou, Guangdong 510630, P.R. China
}

Received December 10, 2017; Accepted March 1, 2018

DOI: $10.3892 / \mathrm{etm} .2018 .6291$

\begin{abstract}
Gestational diabetes mellitus (GDM) is a metabolic disease with symptoms of hyperglycemia, insulin resistance and fetal maldevelopment. Evidence has indicated that fibroblast growth factor (FGF)-21 is a multifunctional protein and exhibits potential therapeutic value for metabolic diseases. The present study investigated the diagnostic value of FGF-21 serum levels in patients with GDM $(n=50)$ and age-matched healthy individuals $(n=50)$. It was demonstrated that the gene and protein expression levels of FGF-21 were downregulated in adipose cells in patients with GDM compared with those in healthy individuals. The results also indicated that the serum levels of FGF-21 were downregulated in patients with GDM compared with those in healthy individuals. In addition, it was demonstrated that blood glucose and blood pressure were higher in patients with GDM compared with those in healthy individuals. GDM patients had a markedly higher insulin resistance and glucose tolerance than healthy individuals. However, GDM patients had significantly lower serum levels of insulin than healthy individuals. It was observed that the serum levels of FGF-21 were positively correlated with those of glucose in GDM patients. In conclusion, these results indicate that decreased FGF-21 levels are associated with the risk of GDM, suggesting that FGF-21 may be a potential diagnostic factor for GDM.
\end{abstract}

\section{Introduction}

Gestational diabetes mellitus (GDM) is defined as diabetes during pregnancy and is the most common pregnancy-associated complication (1). Symptoms of GDM include hyperglycemia, glucose intolerance, insulin resistance and

Correspondence to: Professor Chengfang $\mathrm{Xu}$, Department of Obstetrics and Gynecology, The Third Affiliated Hospital, Sun Yat-sen University, 600 Tianhe Road, Guangzhou, Guangdong 510630, P.R. China

E-mail: xuchengfanggz@163.com

Key words: gestational diabetes mellitus, fibroblast growth factor-21, insulin resistance, glucose intolerance fetal maldevelopment (2). A previous study has indicated that GDM may increase the risk of type II diabetes mellitus and cardiovascular disease (3). Studies also suggested that GDM poses a great threat for the fetal and maternal safety during pregnancy (4-6). The International Association of Diabetes in Pregnancy Study Group criteria for GDM have been recommended by World Health Organization (7). To date, various diagnostic methods have been suggested based on the understanding of the molecular mechanisms of the pathogenesis of this disease (8-10). Therefore, investigation of potentially implicated cytokines may be beneficial for diagnosing patients with GDM.

Fibroblast growth factor-21 (FGF-21) is an atypical member of the family of FGFs, and is regarded as a multifunctional cytokine (11). A study has indicated that FGF-21 is also a multifunctional protein predominantly secreted by adipose tissue, pancreas and liver, and has been regarded as a polypeptide with efficacy in the treatment of metabolic disorders $(12,13)$. FGF21 is produced in the liver and has a crucial role in regulating glucose and lipid metabolism, as well as maintaining energy homeostasis; it has been implicated in the regulation of the endocrine metabolism and various chronic diseases occurs via regulated metabolic processes, including glucose and lipid metabolism (14). Wang et al (15) have indicated that the serum concentration of FGF-19 and -21 in maternal patients with gestational diabetes mellitus is associated with insulin resistance, adiponectin and a history of polycystic ovary syndrome. However, the diagnostic value of the serum levels of FGF-21 in gestational diabetes mellitus patients has remained to be fully elucidated.

The present study assessed the potential diagnostic value of human FGF-21 for GDM and its possible association with insulin resistance and glucose tolerance in affected patients. The results demonstrated that the serum levels of FGF-21 were significantly downregulated in GDM patients, which may be associated with glucose and insulin metabolism, and suggest that FGF-21 is a potential diagnostic factor for the diagnosis of GDM.

\section{Materials and methods}

Ethical statement. The protocols were approved by Ethics Committee of The Third Affiliated Hospital of Sun Yat-sen University (Guangzhou, China). Patients and healthy 
individuals were required to provide written informed consent. The authors had no access to any information that could identify the individual participants during or after data collection.

Patients and healthy volunteers. A total of 50 patients with GDM and 50 age-matched healthy individuals were enrolled in The Third Affiliated Hospital of Sun Yat-sen University between May 2013 and June 2014 in the present study. The mean age was 28.3 and 28.6 years in the GDM group and healthy individuals, respectively. There was a difference in mean BMI values between the GDM patients and healthy individuals. The mean duration of follow-up in the GDM group was 6 months (Table I). The inclusion criteria were as follows: i) Gestation period of 4-8 months; ii) no history of diabetes mellitus, and no presence of renal failure, intake of medications, ischemic heart disease, heart failure or pancreatic disease.

ELISA. The serum levels of FGF-21 in patients with GDM and healthy volunteers were detected using an ELISA kit (cat. no. KA1849; Abnova, Taipei, Taiwan) according to the manufacturer's protocols. Finally, the serum concentration levels of FGF-21 were measured with an ELISA microplate reader (Bio-Rad Laboratories, Inc., Hercules, CA, USA) at $450 \mathrm{~nm}$.

Reverse transcription-quantitative polymerase chain reaction (RT-qPCR). Total RNA was extracted from adipocytes using by using an RNAeasy Mini Kit (Qiagen, Hilden, Germany) according to the manufacturer's protocols. Reverse transcription was performed at $42^{\circ} \mathrm{C}$ for $2 \mathrm{~h}$ in a total of $50 \mu \mathrm{l}$ containing 50 ng RNA, $2 \mu 1$ primers, $5 \mu \mathrm{l}$ dNTP, $2 \mu 1$ RT-buffer, and $1 \mu 1$ reverse transcriptase. All of the forward and reverse primers were synthesized by Invitrogen (Thermo Fisher Scientific, Inc., Waltham, MA, USA) with the following sequences: FGF-21 forward, 5'-CTGCTGGGGGTCTACCAAG-3' and reverse, 5'-CTGCGCCTACCACTGTTCC-3'; $\beta$-actin forward, 5'-CATCTCTTGCTCGAAGTCCA-3' and reverse, 5'-ATC ATGTTTGAGACCTTCAACA-3'. PCR amplification was performed using the following program: Initial denaturation at $94^{\circ} \mathrm{C}$ for $2 \mathrm{~min}$, followed by 45 cycles of $95^{\circ} \mathrm{C}$ for $30 \mathrm{sec}$, annealing at $56^{\circ} \mathrm{C}$ for $30 \mathrm{sec}$ and $72^{\circ} \mathrm{C}$ for $10 \mathrm{~min}$. The reaction was performed in a volume of $20 \mu \mathrm{l}$ containing $50 \mathrm{ng}$ genomic cDNA, $200 \mu \mathrm{M}$ deoxynucleoside triphosphate, 2.5 units of Taq DNA polymerase (Takara Biotechnology Co., Ltd., Dalian, China) and $200 \mu \mathrm{M}$ primers using PCR (iQ5 Real Time PCR System; Bio-Rad Laboratories, Inc.). Relative mRNA expression levels were calculated via the $2^{-\Delta \Delta \mathrm{Cq}}$ method (16). The results are expressed as the ratio of the $\beta$-actin control.

Western blot analysis. Adipose cells were isolated from GDM and healthy volunteers as described previously (17) and lysed in radioimmunoprecipitation assay buffer [mammalian protein extraction reagent (PER) for the cells and tissue PER for the tissues; Thermo Fisher Scientific, Inc.] followed by homogenization at $4^{\circ} \mathrm{C}$ for $0 \mathrm{~min}$. The protein concentration was measured with a bicinchoninic protein assay kit (Thermo Fisher Scientific, Inc.). A total of $10 \mu \mathrm{g}$ protein extract was electrophoresed on $12.5 \%$ SDS-PAGE and then transferred to polyvinylidene difluoride membranes (EMD Millipore, Billerica, MA, USA). The membranes were incubated in blocking buffer (5\% BSA; Sigma-Aldrich; Merck KGaA) prior
Table I. Characteristics of GDM patients compared with healthy volunteers.

\begin{tabular}{lcc}
\hline Parameter & $\begin{array}{c}\text { GDM } \\
\text { patients }\end{array}$ & $\begin{array}{c}\text { Healthy } \\
\text { volunteers }\end{array}$ \\
\hline Mean age (years) & 28.3 & 28.6 \\
No. of patients & $(22.5-35.2)$ & $(23.2-34.5)$ \\
Follow-up time (months) & 50 & 50 \\
Blood glucose (mmol/l) & 6 & 6 \\
Blood pressure (mmHg) & $9.3 \pm 0.9$ & $6.5 \pm 0.7^{\mathrm{a}}$ \\
Insulin concentration $(\mathrm{mmol} / \mathrm{l})$ & $124 \pm 14$ & $120 \pm 16$ \\
Body mass index $\left(\mathrm{kg} / \mathrm{m}^{2}\right)$ & $9.2 \pm 1.2$ & $14.9 \pm 2.5^{\mathrm{a}}$ \\
\hline
\end{tabular}

${ }^{\mathrm{a}} \mathrm{P}<0.01$ vs. GDM group. GDM, gestational diabetes mellitus.

to incubation with primary antibodies at $4^{\circ} \mathrm{C}$ overnight. The following primary rabbit anti-human antibodies were used in the immunoblotting assays: FGF-21 (1:1,000 dilution; cat. no. ab171941) and GAPDH (1:1,500 dilution; cat. no. ab9485; both from Abcam, Cambridge, MA, USA). After the incubation, the membrane was washed three times in Tris-buffered saline containing Tween-20 (TBST) and incubated with horseradish peroxidase (HRP)-conjugated goat anti-rabbit immunoglobulin monoclonal antibody (cat. no. PV-6001; Zhongshan Goldenbridge-Bio, Beijing, China) for $1 \mathrm{~h}$ at $37^{\circ} \mathrm{C}$. After three washes in TBST, the membrane was developed using a chemiluminescence assay system (cat no. 17-677; Roche Diagnostics, Basel, Switzerland) and exposed to Kodak film (Eastman-Kodak, Rochester, NY, USA). Densitometric quantification of the immunoblot data was performed by using Quantity-One software 1.20 (Bio-Rad Laboratories, Inc.).

Immunohistochemistry staining. Immunohistochemical analysis was performed as described previously (18). Paraffin-embedded $4-\mu \mathrm{m}$ in adipose tissue sections were prepared for further analysis. The paraffin sections were incubated with hydrogen peroxide $(3 \%)$ for $15 \mathrm{~min}$ at $37^{\circ} \mathrm{C}$ and then hydrated in a decreasing series of ethanols. Antigen retrieval was performed using an antigen retrieval kit (cat. no. ab93684; Abcam). Subsequently, 5\% bovine serum albumin (Sigma-Aldrich; Merck $\mathrm{KGaA}$ ) was used to block non-specific binding at $37^{\circ} \mathrm{C}$ for $2 \mathrm{~h}$ and tissue sections were incubated with the abovementioned primary antibody to FGF-21 (1:1,000 dilution) at $4^{\circ} \mathrm{C}$ for $12 \mathrm{~h}$. All sections were washed three times and incubated with the abovementioned HRP-conjugated secondary antibody (1:10,000; cat. no. PV-6001; OriGene Technologies, Inc., Beijing, China) for $12 \mathrm{~h}$ at $4^{\circ} \mathrm{C}$. Diaminobenzidine (Sigma-Aldrich; Merck KGaA) was incubated for $5 \mathrm{~min}$ at $37^{\circ} \mathrm{C}$ used to visualize the antibodies at room temperature for $10 \mathrm{sec}$. Positive signals were detected in six random fields of view under an inverted light microscope (Olympus Corporation, Tokyo, Japan) and images were captured.

Glucose tolerance and insulin resistance test. GDM patients and healthy individuals were fasted for $6 \mathrm{~h}$ and orally given 
A

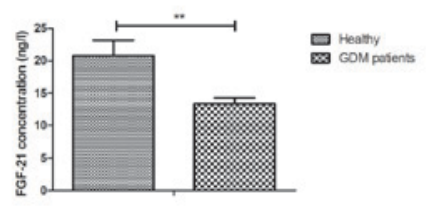

D
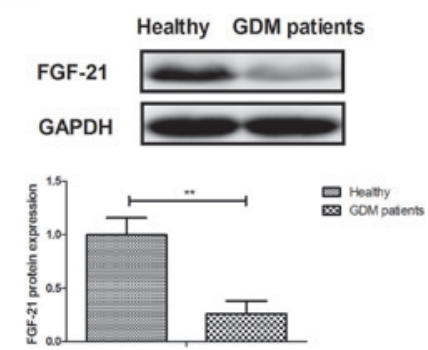

B

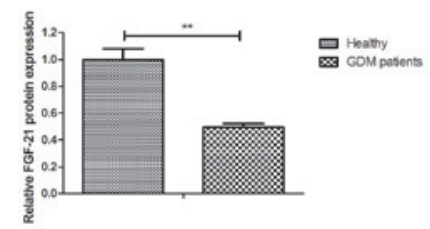

E

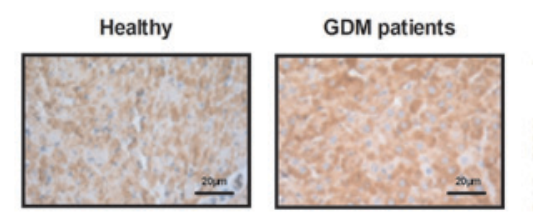

C
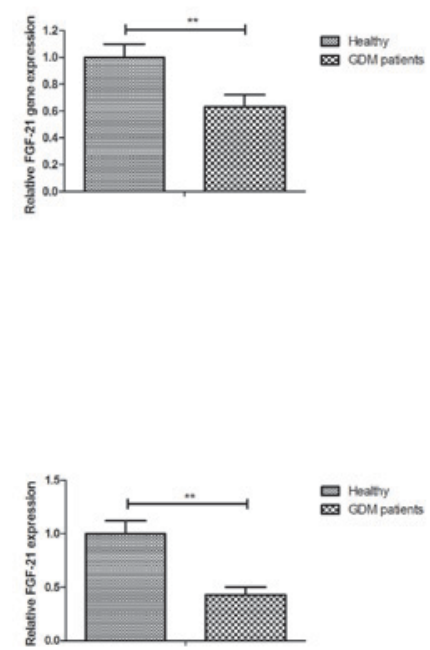

Figure 1. Analysis serum levels of FGF-21 in GDM patients. (A) Serum levels of FGF-21 in GDM patients and healthy individuals. (B) Protein levels of FGF-21 in adipocytes were downregulated in clinical patients compared with those in healthy volunteers. (C) FGF-21 mRNA expression levels are downregulated in adipocytes of GDM patients compared with those in healthy volunteers. (D) Protein expression levels of FGF-21 were downregulated in patients with GDM in adipocytes compared with those in healthy individuals. (E) Expression levels of FGF-21 in adipose tissue between GDM patients and healthy individuals determined by immunohistochemistry. ${ }^{* *} \mathrm{P}<0.01$. FGF, fibroblast growth factor; GDM, gestational diabetes mellitus.

glucose at a dose of $2.0 \mathrm{~g} / \mathrm{kg}$ for the glucose tolerance test. The blood glucose concentration was analyzed with an ACCU-CHEK Advantage glucometer (Roche Diagnostics). The glucose tolerance test results were recorded at baseline and after glucose injection ( $0,15,45,75$ and $105 \mathrm{~min})$. For the insulin tolerance test, all participants were intraperitoneally injected insulin at $0.75 \mathrm{U} / \mathrm{kg}$ body weight. All participants were intraperitoneally injected with insulin $(1 \mathrm{mU} / \mathrm{kg})$ after a $0,15,30,45,60,75$ and 90 min fast and the blood glucose concentration was measured at baseline and after insulin injection (15, 30, 60, 90 and $120 \mathrm{~min})$.

Statistical analysis. Values are expressed as the mean \pm standard error of the mean or median (interquartile range) Data were analyzed by Student's t-test or analysis of variance, followed by Dunnett's post-hoc test using GraphPad Prism software 5.0 (GraphPad Inc., La Jolla, Ca, USA). The differences in regression coefficients between models were compared by Student's t-test. $\mathrm{P}<0.05$ was considered to indicate a statistically significant difference.

\section{Results}

Serum levels of FGF-21 in GDM patients. First, FGF-21 expression was detected in the serum and cells of GDM patients. As presented in Fig. 1A, the serum levels of FGF-21 were downregulated in patients with GDM compared with those in healthy individuals. Furthermore, FGF-21 protein was downregulated in adipocytes isolated from GDM patients compared with those of healthy volunteers (Fig. 1B). Gene expression analysis also indicated that FGF-21 mRNA levels were downregulated in adipocytes isolated from GDM patients compared with those in healthy volunteers (Fig. 1C). As presented in Fig. 1D, the protein expression levels of FGF-21 in adipocytes were markedly downregulated in patients with GDM compared with those in healthy individuals. Immunohistochemistry indicated that the expression levels of FGF-21 were also decreased in adipose tissue from GDM patients compared with those in healthy individuals (Fig. 1E). These results suggest that FGF-21 is downregulated in serum and adipocytes of patients with GDM.

Analysis of glucose and insulin metabolism in GDM patients. Next, the glucose and insulin metabolism was assessed in GDM patients and healthy volunteers. The results indicated that GDM patients presented with a higher blood glucose concentration than healthy volunteers (Fig. 2A). Furthermore, it was observed that insulin levels were slightly decreased in GDM patients (Fig. 2B). It was also revealed that GDM patients had significantly higher glucose intolerance and insulin resistance than healthy individuals (Fig. 2C and D). These results indicate that compared to healthy individuals, GDM patients exhibited disorders in glucose and insulin metabolism.

Association of serum levels of FGF-21 with glucose and insulin metabolism in GDM patients. The correlation of the levels of FGF-21 with parameters of GDM was then assessed. As presented in Fig. 3A, the plasma levels of FGF-21 were positively correlated with the blood glucose levels in GDM patients. Furthermore, the plasma levels of FGF-21 were positively correlated with the concentration of insulin in GDM patients (Fig. 3B). These results indicate that FGF-21 is associated with the serum levels of glucose as well as with the insulin levels in GDM patients.

Association between serum levels of FGF-21 and prognosis of GDM patients. Finally, the association between serum levels of FGF-21 and the prognosis of GDM patients was analyzed. The results indicated that the serum levels of FGF-21 were upregulated in GDM patients after the end of 
A

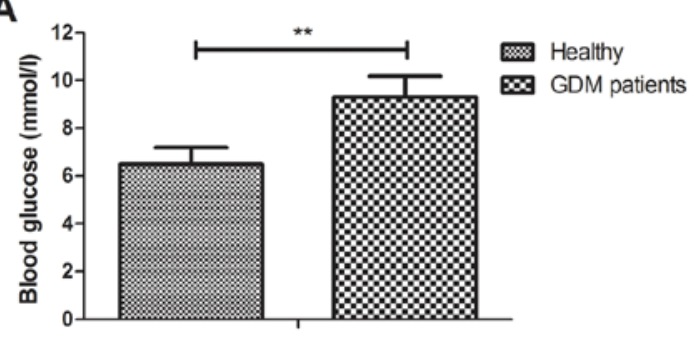

C

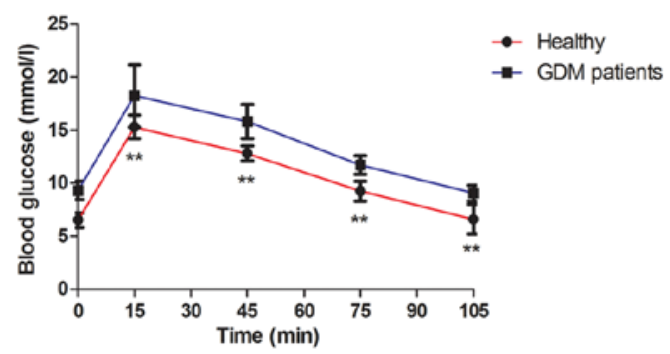

B

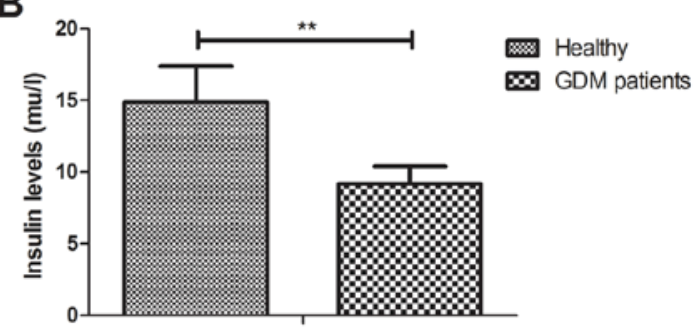

D

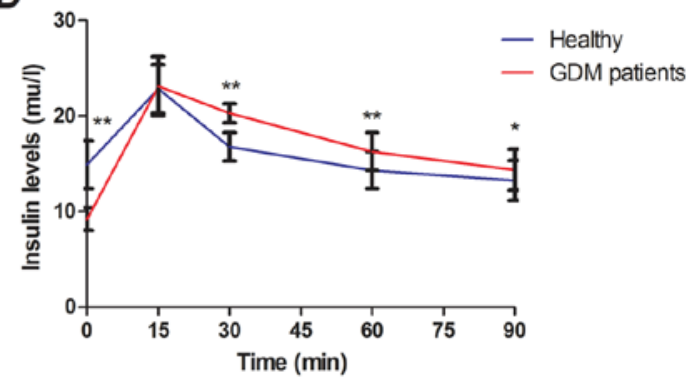

Figure 2. Analysis of glucose and insulin metabolism in GDM patients. (A) GDM patients had a higher blood glucose concentration than healthy volunteers. (B) Insulin levels were decreased in GDM patients compared with those in healthy volunteers. (C) GDM patients had a higher glucose tolerance than healthy volunteers. (D) GDM patients had a higher insulin resistance than healthy individuals. " $\mathrm{P}<0.05$ and ${ }^{* * *} \mathrm{P}<0.01$ vs. GDM. GDM, gestational diabetes mellitus.

A

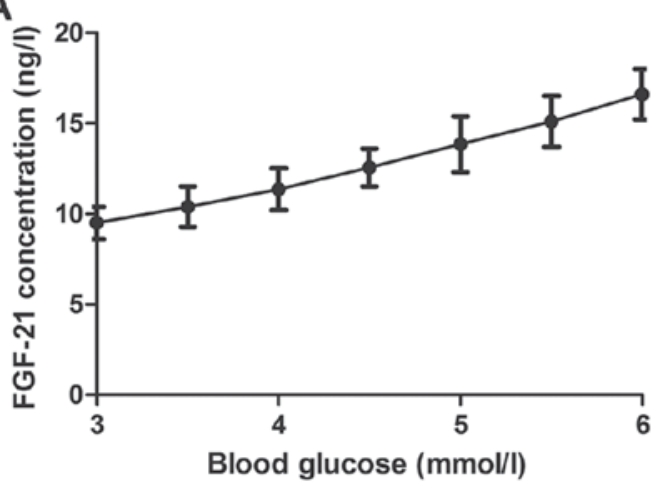

B

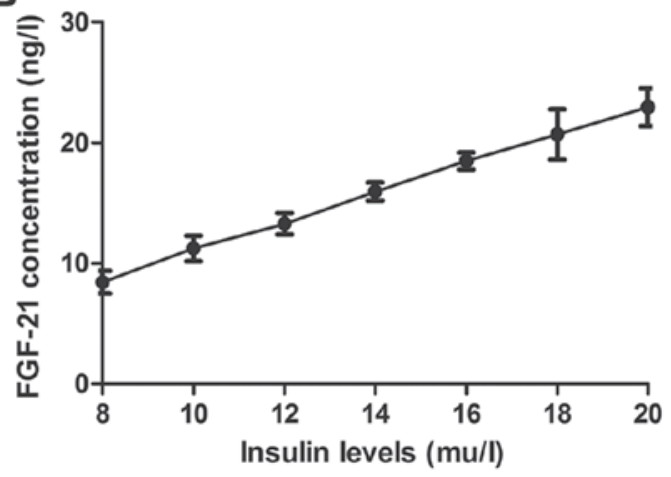

Figure 3. Analysis of the association of serum levels of FGF-21 with glucose and insulin metabolism in GDM patients. (A) Serum levels of FGF-21 are positively correlated with the concentration of blood glucose in GDM patients. (B) Serum levels of FGF-21 are positively correlated with insulin levels in GDM patients. FGF, fibroblast growth factor; GDM, gestational diabetes mellitus.

pregnancy (Fig. 4A). The serum levels of FGF-21 were also associated with the clinical stage of GDM patients (Fig. 4B). These results indicate that FGF-21 may be a prognostic indicator in patients with GDM.

\section{Discussion}

FGF-21 has been reported as a novel hepatoprotective substance (19), and has been identified as a momentous controller and regulator of glucose and lipid metabolism, as well as long-term energy balance (20,21). A study has indicated that attenuation of FGF signaling in mouse $\beta$-cells leads to diabetes (22). However, the role of FGF-21 in the diagnosis of GDM has remained to be fully elucidated. The present study investigated the diagnostic value of FGF-21 in patients with suspected GDM. The results indicated that the serum levels of FGF-21 are downregulated in GDM patients. It was demonstrated that the serum levels of FGF-21 are correlated with insulin resistance and glucose tolerance in clinical GDM patients.

Studies have suggested that the effects of FGF-21 on metabolic hormones to regulate energy metabolism are essential for human vascular endothelial cells $(23,24)$. Wang et al $(25)$ have indicated that FGF-21 is positively associated with atrial fibrosis in atrial fibrillation patients with rheumatic heart disease. The present study reported that GDM patients exhibit a disorder in glucose and insulin metabolism compared to healthy individuals. Chen et al (26) have indicated that plasma insulin and Helicobacter pylori outer membrane protein A are independent factors influencing plasma FGF-21 levels, and due to its role in the pathogenesis of insulin resistance and type 2 diabetes mellitus, FGF-21 is a potential diagnostic factor. The present results indicate that the serum levels of FGF-21 are positively correlated with the concentration of blood glucose and insulin 
A

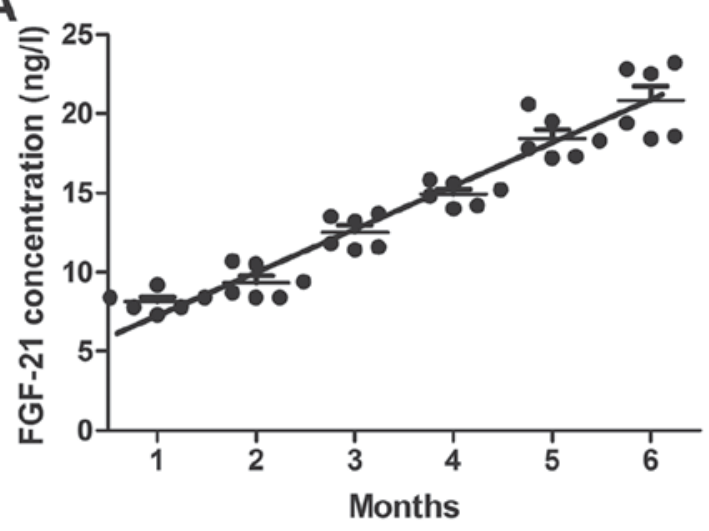

B

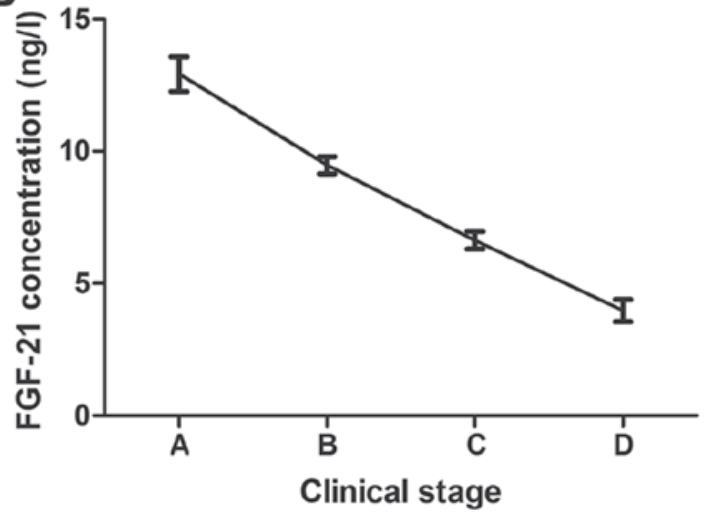

Figure 4. Analysis the correlation between serum levels of FGF-21 and the prognosis of GDM patients. (A) Serum levels of FGF-21 are upregulated in GDM patients with the recovery time progressing $(\mathrm{r}=0.412)$. (B) Serum levels of FGF-21 are associated with the clinical stage of GDM patients ( $\mathrm{r}=-0.376)$. FGF, fibroblast growth factor; GDM, gestational diabetes mellitus.

metabolism in GDM patients. The results also demonstrated that FGF-21 expression is significantly different between the GDM and healthy group. However, the association between FGF-21 and BMI should be further established in future studies.

FGF-21 alleviates diabetes-associated vascular complications by inhibiting nuclear factor- $\kappa \mathrm{B} / \mathrm{NACHT}, \mathrm{LRR}$ and PYD domains-containing protein 3 inflammasome-mediated inflammation (27). A previous study also suggested that a high dose of polyethylene glycol-conjugated FGF-21 $(500 \mathrm{mg} / \mathrm{kg})$ at the start, followed by a low maintenance dose results has favorable effects by controlling the glycolipid metabolic balance, providing a novel method for the management of diabetes (28). The results of the present study indicated that the serum levels of FGF-21 are associated with the prognosis of GDM patients, suggesting that FGF-21 is a potential prognostic marker in patients with GDM. Chen et al (26) have demonstrated that FGF-21 not only increases insulin secretion and insulin content in diabetic islets, but also protects $\beta$-cells from apoptosis via the activation of extracellular signal-regulated kinase $1 / 2$ and Akt signaling pathways. The present study reported that the insulin concentration in GDM patients returns to normal levels after the end of the pregnancy (within 3 months). However, the control group was not pregnant, which is a limitation of the present study. Further studies should thus incorporate a pregnant control.

In conclusion, the present study suggests that downregulation of FGF-21 may be associated with the risk of GDM. Of note, the results indicate that FGF-21 may be a diagnostic and prognostic indicator in patients with GDM. However, further studies should be performed in large populations to assess the association between FGF-21 with impaired glucose metabolism.

\section{Acknowledgements}

Not applicable.

\section{Funding}

The present study was financially supported by the Development of Science and Technology of the Department of Science and Technology of Guangzhou, China (grant no. 201704020170).

\section{Availability of data and materials}

The datasets used and/or analyzed during the current study are available from the corresponding author on reasonable request.

\section{Authors' contributions}

CX designed the present study. ZH, PL and XL performed all experiments and analyzed the data in the present study.

\section{Ethical approval and consent to participate}

The protocols were approved by the Ethics Committee of The Third Affiliated Hospital of Sun Yat-sen University (Guangzhou, China). All patients and healthy individuals were required to provide written informed consent.

\section{Consent for publication}

Not applicable.

\section{Competing interests}

The authors declare that they have no competing interests.

\section{References}

1. Popova P, Castorino K, Grineva EN and Kerr D: Gestational diabetes mellitus diagnosis and treatment goals: measurement and measures. Minerva Endocrinol 41: 421-432, 2016.

2. Khalafallah A, Phuah E, Al-Barazan AM, Nikakis I, Radford A, Clarkson W, Trevett C, Brain T, Gebski V and Corbould A: Glycosylated haemoglobin for screening and diagnosis of gestational diabetes mellitus. BMJ Open 6: e011059, 2016.

3. Hopmans TE, van Houten C, Kasius A, Kouznetsova OI, Nguyen LA, Rooijmans SV, Voormolen DN, van Vliet EO, Franx A and Koster MP: Increased risk of type II diabetes mellitus and cardiovascular disease after gestational diabetes mellitus: A systematic review. Ned Tijdschr Geneeskd 159: A8043, 2015 (In Dutch).

4. Wei Y, Yang H, Zhu W, Li H, Yan J and Zhang C: International association of diabetes and pregnancy study group criteria is suitable for gestational diabetes mellitus diagnosis: Further evidence from China. Chin Med J (Engl) 127: 3553-3556, 2014. 
5. Kalter-Leibovici O, Freedman LS, Olmer L, Liebermann N, Heymann A, Tal O, Lerner-Geva L, Melamed N and Hod M: Screening and diagnosis of gestational diabetes mellitus: Critical appraisal of the new International Association of Diabetes in pregnancy study group recommendations on a national level Diabetes Care 35: 1894-1896, 2012.

6. Tan PC, Aziz AZ, Ismail IS and Omar SZ: Gamma-glutamyltransferase, alanine transaminase and aspartate transaminase levels and the diagnosis of gestational diabetes mellitus. Clin Biochem 45: 1192-1196, 2012.

7. Sagili H, Kamalanathan S, Sahoo J, Lakshminarayanan S, Rani R, Jayalakshmi D and Kumar KT: Comparison of different criteria for diagnosis of gestational diabetes mellitus. Indian J Endocrinol Metab 19: 824-828, 2015.

8. Askari G, Iraj B, Salehi-Abargouei A, Fallah AA and Jafari T: The association between serum selenium and gestational diabetes mellitus: A systematic review and meta-analysis. J Trace Elem Med Biol 29: 195-201, 2015.

9. Amin M, Suksomboon N, Poolsup N and Malik O: Comparison of glyburide with metformin in treating gestational diabetes mellitus: A systematic review and meta-analysis. Clin Drug Investig 35: 343-351, 2015.

10. Zhuo Z, Wang A and Yu H: Effect of metformin intervention during pregnancy on the gestational diabetes mellitus in women with polycystic ovary syndrome: a systematic review and meta-analysis. J Diabetes Res 2014: 381231, 2014.

11. Durovcová V, Marek J, Hána V, Matoulek M, Zikán V, Haluzíková D, Kaválková P, Lacinová Z, Krsek M and Haluzík M: Plasma concentrations of fibroblast growth factors 21 and 19 in patients with Cushing's syndrome. Physiol Res 59: 415-422, 2010.

12. Eto K: FGF-21, a newcomer in the field of hypertension research J Hum Hypertens 27: 343-344, 2013.

13. Reinehr T, Woelfle J, Wunsch R and Roth CL: Fibroblast growth factor 21 (FGF-21) and its relation to obesity, metabolic syndrome, and nonalcoholic fatty liver in children: A longitudinal analysis. J Clin Endocrinol Metab 97: 2143-2150, 2012.

14. Zhang F, Yu L, Lin X, Cheng P, He L, Li X, Lu X, Tan Y, Yang H, Cai L and Zhang C: Minireview: Roles of fibroblast growth factors 19 and 21 in metabolic regulation and chronic diseases. Mol Endocrinol 29: 1400-1413, 2015.

15. Wang D, Zhu W, Li J, An C and Wang Z: Serum concentrations of fibroblast growth factors 19 and 21 in women with gestational diabetes mellitus: Association with insulin resistance, adiponectin, and polycystic ovary syndrome history. PloS One 8: e81190, 2013

16. Livak KJ and Schmittgen TD: Analysis of relative gene expression data using real-time quantitative PCR and the 2(-Delta Delta C(T)) method. Methods 25: 402-408, 2001.

17. Williams KJ, Picou AA, Kish SL, Giraldo AM, Godke RA and Bondioli KR: Isolation and characterization of porcine adipose tissue-derived adult stem cells. Cells Tissues Organs 188: 251-258, 2008
18. Selinger CI, Li BT, Pavlakis N, Links M, Gill AJ, Lee A, Clarke S, Tran TN, Lum T, Yip PY, et al: Screening for ROS1 gene rearrangements in non-small-cell lung cancers using immunohistochemistry with FISH confirmation is an effective method to identify this rare target. Histopathology 70: 402-411, 2017.

19. Cariello M and Moschetta A: Fibroblast growth factor 21: A new liver safeguard. Hepatology 60: 792-794, 2014

20. Suomalainen A, Elo JM, Pietiläinen KH, Hakonen AH, Sevastianova K, Korpela M, Isohanni P, Marjavaara SK, Tyni T, Kiuru-Enari S, et al: FGF-21 as a biomarker for muscle-manifesting mitochondrial respiratory chain deficiencies: A diagnostic study. Lancet Neurol 10: 806-818, 2011.

21. Lin Z, Wu Z, Yin X, Liu Y, Yan X, Lin S, Xiao J, Wang X, Feng W and Li X: Serum levels of FGF-21 are increased in coronary heart disease patients and are independently associated with adverse lipid profile. PloS One 5: e15534, 2010.

22. Hart AW, Baeza N, Apelqvist A and Edlund H: Attenuation of FGF signalling in mouse beta-cells leads to diabetes. Nature 408 : $864-868,2000$.

23. Dushay J, Chui PC, Gopalakrishnan GS, Varela-Rey M, Crawley M, Fisher FM, Badman MK, Martinez-Chantar ML and Maratos-Flier E: Increased fibroblast growth factor 21 in obesity and nonalcoholic fatty liver disease. Gastroenterology 139 456-463, 2010.

24. Hotta Y, Nakamura H, Konishi M, Murata Y, Takagi H, Matsumura S, Inoue K, Fushiki T and Itoh N: Fibroblast growth factor 21 regulates lipolysis in white adipose tissue but is not required for ketogenesis and triglyceride clearance in liver. Endocrinology 150: 4625-4633, 2009.

25. Wang R, Yi X, Li X and Jiang X: Fibroblast growth factor-21 is positively associated with atrial fibrosis in atrial fibrillation patients with rheumatic heart disease. Int J Clin Exp Pathol 8: 14901-14908, 2015.

26. Chen WW, Li L, Yang GY, Li K, Qi XY, Zhu W, Tang Y, Liu H and Boden G: Circulating FGF-21 levels in normal subjects and in newly diagnose patients with Type 2 diabetes mellitus. Exp Clin Endocrinol Diabetes 116: 65-68, 2008.

27. Liu MH: FGF-21 alleviates diabetes-associated vascular complications: Inhibiting NF-kB/NLRP3 inflammasome-mediated inflammation? Int J Cardiol 185: 320-321, 2015.

28. Xu P, Zhang Y, Song L, Khoso MH, Li J, Jiang X, He J, Li J, Ma X, Ren G and Li D: Efficacy of a combination of high and low dosage of PEGylated FGF-21 in treatment of diabetes in $\mathrm{db} / \mathrm{db}$ mice. Biomed Pharmacother 84: 97-105, 2016. 